\title{
Combining Ability Analysis for Yield and Quality Traits in Tomato (Solanum lycopersicum L.)
}

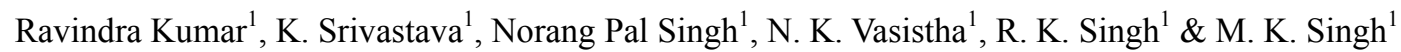 \\ ${ }^{1}$ Department of Genetics \& Plant Breeding, Institute of Agricultural Sciences, Banaras Hindu University Varanasi, \\ India
}

Correspondence: Ravindra Kumar, Department of Genetics \& Plant Breeding, Institute of Agricultural Sciences, Banaras Hindu University Varanasi, India. E-mail: godwalravindra@gmail.com

$\begin{array}{lc}\text { Received: November 1, } 2012 & \text { Accepted: November 16, } 2012 \quad \text { Online Published: January 15, } 2013 \\ \text { doi:10.5539/jas.v5n2p213 } & \text { URL: http://dx.doi.org/10.5539/jas.v5n2p213 }\end{array}$

\begin{abstract}
Thirteen parental lines were crossed in line $\mathrm{X}$ tester fashion comprising 10 lines and 3 testers at vegetable farm Banaras Hindu University Varanasi to estimate combining ability in tomato for fruit yield, yield components and fruit quality traits. $F_{1}$ and parents were grown in towards four and half meters in randomized block design with three replications during winter-2010 involvement of both additive and non additive gene action was operated for the control of fruits per plant, fruit weight and average fruit weight. All the fruit quality characters like, total soluble solids (TSS), titratable acidity, ascorbic acid and lycopene of the fruit were governed by non additive gene action. In most of the traits, over-dominance was predominant. The analysis of components of genetic variance for yield components showed that the main part of genetic variance was due to additive effect. Estimation of general combining ability (GCA) for yield and earliness showed that Pant T-3 had the highest GCA for increasing yield and Punjab Upma had the highest GCA for both earliness and average fruit weight. Cross combination CO-3 X Azad T-5 exhibit significant specific combining ability (SCA) for the most of desirable traits among all cross combinations.
\end{abstract}

Keywords: combining ability, fruit, character, desirable and components

\section{Introduction}

Tomato (Lycopersicon esculentum Mill.) is the most widely grown vegetable of the World. India is the second largest tomato producer of the world after China, accounting for about $11 \%$ of the world tomato production (Indian Horticulture Database, 2011). During 2010-11, the area and production of tomato in India was about 0.865 million hectare and 16.82 million tonnes, with an average productivity 19.6 tonnes per hecatre (Indian Horticulture Database, 2011). Tomato is universally treated as 'Protective Food' since it is a rich of minerals, vitamins, antioxidants and organic acids. The nutrition importance of the tomato indicates there is need to formulate breeding programme and to develop cultivars rich in lycopene, processing traits with high quality of fruit as well as yield. Recent studies indicate that lycopene that give the ripe tomato its bright red colour, is a very effective natural antioxidant and quencher of free radicals (Simon, 1992). Lycopene is especially efficient in neutralizing Reactive Oxygen Species (ROS). These properties of lycopene are due to its unique chemical structure, a very long chain of conjugated double bonds. Efforts are being made to increase its productivity by developing superior varieties. The entire genetic variability observed in the analysis for each trait was partitioned into its components, i.e. GCA and SCA as defined by Sprague (1966) and reciprocal effects as sketched by Griffing (1956). They stated that GCA effects were due to additive type of gene action and SCA effects were due to non-additive (dominant or epistatic) gene action. Several studies of combining ability for yield components are available in many species. Some researchers found the predominancy of GCA to be more important than that of SCA (Khan et al., 1991; Yaqoob et al., 1997), while others suggested that SCA was more important (Ortiz, 2004; Biswas et al., 2005).

\section{Methods and Materials}

The present investigations were conducted during winter-2010 at Vegetable Research Farm, Institute of Agricultural Sciences, Banaras Hindu University Varanasi, which is situated at $25^{\circ}$ North latitude, $83^{\circ}$ East longitude at a mean altitude of 80.71 meters above the sea level, receiving annual rainfall of $110 \mathrm{~cm}$ and sandy loam type soil. 


\subsection{Experimental Material}

The experimental material comprised ten genetically divers lines (H-24, DT-2, CO-3, Punjab Upma, Pant T-3, H-86, Selection-7, NDTVR-60, Fla-7171 \& Kashi Amrit) and three testers (Floradade, Kashi Sharad \& Azad T-5) along with their $30 \mathrm{~F}_{1}$ hybrids developed by crossing them in a line $\mathrm{X}$ tester mating design (Kempthorne, 1957). All the 43 genotypes ( 13 parents and $30 \mathrm{~F}_{1}$ hybrids) were evaluated; the seedlings were transplanted in a randomized block design with three replications at the spacing of $60 \mathrm{~cm}$ between rows and $45 \mathrm{~cm}$ between plants. Recommended cultural practices and plant protection measures were followed. The observation were recorded for eleven traits i.e. plant height, day to $50 \%$ flowering, number of primary branches, number of fruits per plant, average fruit weight, fruit per cluster, total yield per plant, TSS, ascorbic acid, titratable acidity \& lycopene and data were compiled for analysis of variance for all these traits using method suggested by Panse and Sukhatme (1967).

\section{Results and Discussion}

The analysis of variance for combining ability (Table 1) showed the existence of significant variation for eleven characters, indicating a wide range of variability among the genotypes. Highly significant variation due to GCA as well as SCA indicated the importance of additive as well as non-additive types of gene action of inheritance for all the traits except the number of fruits per plant.

Table 1. Analysis of variance for general and specific combining ability in parents and $F_{1} s$ for character studied in tomato

\begin{tabular}{|c|c|c|c|c|c|c|c|c|c|c|c|c|}
\hline Source & D.F. & $\begin{array}{l}\text { Plant } \\
\text { Height }\end{array}$ & $\begin{array}{l}\text { Day to } 50 \% \\
\text { Flowering }\end{array}$ & $\begin{array}{l}\text { No. of } \\
\text { primary } \\
\text { branches }\end{array}$ & $\begin{array}{c}\text { No. of } \\
\text { fruits/plant }\end{array}$ & $\begin{array}{c}\text { Average } \\
\text { fruit weight }\end{array}$ & Fruit/cluster & $\begin{array}{c}\text { Total } \\
\text { yield/plant }\end{array}$ & $\begin{array}{c}\text { Total } \\
\text { Soluble } \\
\text { Solids }\end{array}$ & $\begin{array}{c}\text { Ascorbic } \\
\text { Acid }\end{array}$ & $\begin{array}{l}\text { Titratable } \\
\text { Acidity }\end{array}$ & Lycopene \\
\hline Replicates & 2 & 26.81 & $8.78^{* *}$ & $1.90^{*}$ & $11.39^{*}$ & $32.29^{*}$ & $0.25 *$ & 0.00 & 0.00 & 0.49 & 0.00 & 0.59 \\
\hline Crosses & 29 & $340.87^{* *}$ & $66.23 * *$ & $2.11 * *$ & $18.48^{* *}$ & $53.36 * *$ & $0.77^{* *}$ & $0.43^{* *}$ & $0.91 * *$ & $8.44 * *$ & $0.01 * *$ & $6.45 * *$ \\
\hline Line Effect & 9 & $727.36 * *$ & $125.07^{*}$ & 2.59 & 12.53 & 80.81 & $1.32 *$ & $0.85^{*}$ & 1.26 & $16.71 * *$ & $0.03 * *$ & 9.84 \\
\hline $\begin{array}{l}\text { Tester } \\
\text { Effect }\end{array}$ & 2 & $1123.93 * *$ & 74.42 & 0.59 & 1.97 & 33.59 & 0.77 & 0.18 & 1.10 & 5.92 & 0.02 & 6.00 \\
\hline $\begin{array}{l}\text { Line* } \\
\text { Tester }\end{array}$ & 18 & $60.62 * *$ & $35.90 * *$ & $2.04 * *$ & $23.30^{* *}$ & $41.83 * *$ & $0.49^{* *}$ & $0.24 * *$ & $0.71 * *$ & 4.59 & $0.01 * *$ & $4.81 * *$ \\
\hline Error & 58 & 8.87 & 1.07 & 0.45 & 3.51 & 6.83 & 0.06 & 0.03 & 0.09 & 2.69 & 0.00 & 0.21 \\
\hline Total & 89 & 117.45 & 22.47 & 1.02 & 8.56 & 22.56 & 0.30 & 0.16 & 0.36 & 4.52 & 0.01 & 2.25 \\
\hline
\end{tabular}

\subsection{Effect of GCA}

Nature and magnitude of combining ability effects provide guideline in identifying the better parents and their utilization. The summery of the GCA effects of the parents (Table 2) revealed that none of the parent found to be good general combiner for all the characters. An overall appraisal of gca effects revealed that among parents $\mathrm{H}-$ 24 emerged out as good general combiner for plant height, days to $50 \%$ flowering, fruits per cluster and total yield per plant whereas, line DT-2 traced out good general combiner for days to $50 \%$ flowering, average fruit weight and TSS and CO-3 for days to 50\% flowering and total yield per plant. Among the parents Punjab Upma was found to be good general combiner for plant height, days to $50 \%$ flowering, and total yield per plant. Pant T-3 for days to $50 \%$ flowering and total yield per plant, whereas H-86 for plant height TSS, titratable acidity and lycopene. Selection -7 for number of fruits per plant, average fruit weight, fruits per cluster, ascorbic acid, titratable acidity and lycopene, while NDTVR-60 for days to $50 \%$ flowering, average fruit weight, TSS, titratable acidity and lycopene. Fla-7171 good general combiner for plant height, fruits per cluster and lycopene whereas, Kashi Amrit only for lycopene. Male parent Floradade for plant height and days to $50 \%$ flowering while Kashi Sharad good general combiner for average fruit weight, total yield per plant an lycopene as well as Azad T-5 for plant height, days to 50\% flowering, fruits per cluster, TSS and Lycopene. 
Table 2. General combing ability (GCA) effect of the 13 parents yield and quality traits

\begin{tabular}{|c|c|c|c|c|c|c|c|c|c|c|c|}
\hline Parents & $\begin{array}{l}\text { Plant } \\
\text { Height }\end{array}$ & $\begin{array}{l}\text { Day to } 50 \% \\
\text { Flowering }\end{array}$ & $\begin{array}{c}\text { No. of primary } \\
\text { branches }\end{array}$ & $\begin{array}{c}\text { No. of } \\
\text { fruits/plant }\end{array}$ & $\begin{array}{l}\text { Average fruit } \\
\text { weight }\end{array}$ & fruit/cluster & $\begin{array}{c}\text { Total } \\
\text { yield/plant }\end{array}$ & $\begin{array}{l}\text { Total Soluble } \\
\text { Solids }\end{array}$ & $\begin{array}{l}\text { Ascorbic } \\
\text { Acid }\end{array}$ & $\begin{array}{l}\text { Titratable } \\
\text { Acidity }\end{array}$ & Lycopene \\
\hline $\mathrm{H}-24$ & $3.29 * *$ & $-1.54 * *$ & -0.01 & 0.85 & $-3.60^{* *}$ & $0.48 * *$ & $0.30^{* *}$ & $-0.44 * *$ & 0.24 & $-0.06^{* *}$ & $-0.79^{* *}$ \\
\hline DT-2 & $-2.57^{*}$ & $-3.03 * *$ & 0.01 & -0.75 & $4.36^{* *}$ & -0.02 & $-0.23 * *$ & $0.49^{* *}$ & $-1.40 * *$ & -0.02 & $-1.16^{* *}$ \\
\hline $\mathrm{CO}-3$ & $-9.82 * *$ & $-3.89 * *$ & -0.20 & -0.64 & 0.82 & -0.00 & $0.37^{* *}$ & 0.22 & $-1.62 * *$ & $-0.09 * *$ & $-1.99^{* *}$ \\
\hline $\begin{array}{l}\text { Punjab } \\
\text { Upma }\end{array}$ & $16.91^{* *}$ & $-4.11 * *$ & 0.19 & 0.77 & $-4.23 * *$ & $-0.52 * *$ & $0.20^{* *}$ & -0.17 & -0.61 & $-0.03 *$ & -0.12 \\
\hline Pant T-3 & 0.17 & $-4.19^{* *}$ & $-1.21 * *$ & 0.71 & -0.66 & $-0.35 * *$ & $0.38^{* *}$ & $-0.3^{* *}$ & 0.76 & -0.00 & -0.14 \\
\hline H-86 & $4.36^{* *}$ & $5.48^{* *}$ & -0.23 & $-2.5^{* *}$ & 1.45 & 0.01 & $-0.49^{* *}$ & $0.56^{* *}$ & 1.02 & $0.05^{* *}$ & $0.39^{*}$ \\
\hline Selection-7 & 1.00 & $3.33^{* *}$ & -0.23 & $1.71^{*}$ & $3.01 * *$ & $0.41 * *$ & $-0.18 * *$ & $-0.44 * *$ & $2.55^{* *}$ & $0.04^{* *}$ & $0.89 * *$ \\
\hline NDTVR-60 & $-6.39 * *$ & $2.13^{* *}$ & 0.51 & 0.09 & $2.25^{*}$ & -0.18 & $-0.30^{* *}$ & $0.26^{* *}$ & $-1.62^{* *}$ & $0.08^{* *}$ & $0.92 * *$ \\
\hline FLA7171 & $7.53^{* *}$ & $2.13^{* *}$ & 0.51 & 0.34 & 0.26 & $0.58 * *$ & $-0.13 *$ & -0.14 & -0.01 & 0.01 & $1.24 * *$ \\
\hline Kashi Amrit & $-14.49 * *$ & $3.71 * *$ & 0.63 & -0.59 & $-3.68^{* *}$ & $-0.39^{* *}$ & 0.07 & 0.05 & 0.77 & 0.01 & $0.76^{* *}$ \\
\hline Floradade & $4.41^{* *}$ & $-1.16^{* *}$ & 0.07 & 0.26 & -0.38 & 0.02 & -0.02 & $-0.21 * *$ & -0.36 & $-0.03 * *$ & $-0.52^{* *}$ \\
\hline $\begin{array}{l}\text { Kashi } \\
\text { Sharad }\end{array}$ & $-6.99 * *$ & $1.79 * *$ & -0.16 & -0.02 & $1.20 *$ & $-0.17 * *$ & $0.09 * *$ & 0.05 & 0.49 & 0.01 & $0.24 * *$ \\
\hline Azad T-5 & $2.58 * *$ & $-0.64 * *$ & 0.08 & -0.24 & -0.80 & $0.15^{*}$ & $-0.06 *$ & $0.16^{*}$ & -0.13 & 0.01 & $0.28^{* *}$ \\
\hline
\end{tabular}

\subsection{Effect of SCA}

In case of SCA effects, none of the hybrid exhibited favourable SCA effect for all the characters (Table 3). Significant SCA effects in favourable direction as observed in many crosses for Plant Height (3), Days of 50\% flowering (8), No. of primary branches (1), No. of fruits per plant (3), Average fruit weight (4), Fruit per cluster (3), Total yield per plant (6), TSS (4), Ascorbic Acid (2), Titratable Acidity (2) and Lycopene (6). This result getting support from the finding of Mahendrakar (2004), Dhaliwal et al. (2004), Premalakshme et al. (2006), and Duhan et al. (2005), Hannan et al. (2007), Saleem et al. (2009), Singh et al. (2010).

The magnitude of SCA effects having a vital importance in selecting the cross combinations with higher probability of obtaining desirable transgressive segregants. The range of SCA effects for total yield per plant varied from -0.23 (Selection -7 X Azad T-5) to 0.56 (Punjab Upma X Azad T-5). Twelve hybrids showed significant SCA effects of which seven hybrids attributed towards positive direction for total yield per plant. Out of thirty crosses, the best five specific crosses were Punjab Upma X Azad T-5 (0.56), followed by crosses CO-3 X Floradade (0.40), CO-3 X Azad T-5 (0.27), Pant T-3 X Azad T-5 (0.24) and NDTVR -60 X Floradade (0.23) for total yield per plant (Table 4). The crosses having best specific combination for total yield per plant were obtained either through Punjab Upma X Azad T-5 (good X poor), CO-3 X Floradade (good X poor), CO-3 X Azad T-5 (good X poor), Pant T-3 X Azad T-5 (good X poor) and NDTVR -60 X Floradade (poor X poor) parental combination. The best specific combination for total yield per plant Viz., Punjab Upma X Azad T-5 recorded the desirable significant SCA effects for trait of days to $50 \%$ flowering. The second best cross i.e., CO-3 X Floradade had desirable significant SCA effects for TSS. Whereas, the third best cross CO-3 X Azad T-5 had significant SCA effects for plant height, average fruit weight, fruits per cluster, and lycopene. The forth best specific combination Pant T-3 X Azad T-5 had desirable significant SCA effects for days to $50 \%$ flowering and average fruit weight. The fifth best specific combination NDTVR-60 X Floradade had desirable significant SCA effects for average Fruit weight, ascorbic acid and lycopene. 
Table 3: Specific combining ability (SCA) effect of the $30 \mathrm{~F} 1$ 's yield and quality traits

\begin{tabular}{|c|c|c|c|c|c|c|c|c|c|c|c|}
\hline CROSSES & $\begin{array}{l}\text { PLANT } \\
\text { HEIGHT }\end{array}$ & $\begin{array}{l}\text { DAY OF } 50 \% \\
\text { FLOWERING }\end{array}$ & $\begin{array}{c}\text { NO. OF } \\
\text { PRIMARY } \\
\text { BRANCHES }\end{array}$ & $\begin{array}{c}\text { NO. OF } \\
\text { FRUITS/PLANT }\end{array}$ & $\begin{array}{c}\text { AVERAGE } \\
\text { FRUIT } \\
\text { WEIGHT }\end{array}$ & FRUIT/CLUSTER & $\begin{array}{c}\text { TOTAL } \\
\text { YIELD/PLANT }\end{array}$ & $\begin{array}{c}\text { TOTAL } \\
\text { SOLUBLE } \\
\text { SOLIDS }\end{array}$ & $\begin{array}{l}\text { ASCORBIC } \\
\text { ACID }\end{array}$ & $\begin{array}{c}\text { TITRATABLE } \\
\text { ACIDITY }\end{array}$ & LYCOPENE \\
\hline $\begin{array}{ll}\mathrm{H}-24 & \mathrm{X} \\
\text { FLORADADE } & \end{array}$ & -1.67 & -0.74 & 0.09 & 1.55 & -0.24 & -0.09 & 0.08 & $-0.49 *$ & 0.51 & 0.00 & $0.87^{* *}$ \\
\hline $\begin{array}{l}\text { H-24 X } \quad \text { KASHI } \\
\text { SHARAD }\end{array}$ & 3.32 & 0.91 & -0.11 & $-3.06^{*}$ & $5.30^{* *}$ & -0.10 & $-0.20^{*}$ & 0.14 & -0.44 & 0.01 & -0.19 \\
\hline H-24 X AZAD T-5 & -1.64 & -0.17 & 0.03 & 1.51 & $-5.06^{* *}$ & 0.19 & 0.12 & 0.34 & -0.07 & -0.01 & $-0.68^{*}$ \\
\hline $\begin{array}{ll}\text { DT-2 } & X \\
\text { FLORADADE } & \end{array}$ & -0.86 & -1.13 & 1.21 & -0.51 & -3.46 & $0.51^{*}$ & 0.06 & $-0.72 * *$ & 0.45 & 0.03 & 0.57 \\
\hline $\begin{array}{l}\text { DT-2 X KASHI } \\
\text { SHARAD }\end{array}$ & -1.91 & $-2.02 * *$ & -0.63 & -0.22 & 2.38 & -0.40 & -0.04 & $0.51^{*}$ & 0.76 & 0.04 & $1.19^{* *}$ \\
\hline DT-2 X AZAD T-5 & 2.77 & $3.15^{* *}$ & -0.58 & 0.73 & 1.08 & -0.11 & -0.02 & 0.21 & -1.21 & $-0.07 * *$ & $-1.77 * *$ \\
\hline $\begin{array}{ll}\text { CO-3 } & X \\
\text { FLORADADE } & \end{array}$ & -3.19 & $-1.59^{*}$ & 0.77 & 0.70 & 0.61 & 0.39 & $0.40^{* *}$ & $0.55^{* *}$ & -1.07 & -0.02 & -0.54 \\
\hline $\begin{array}{l}\text { CO-3 X KASHI } \\
\text { SHARAD }\end{array}$ & $-7.29^{* *}$ & $1.58^{*}$ & -0.96 & $4.50^{* *}$ & $-5.28 * *$ & $-0.81 * *$ & -0.14 & $-0.72 * *$ & -0.38 & -0.04 & $-1.88^{* *}$ \\
\hline CO-3 X AZAD T-5 & $10.48^{* *}$ & 0.01 & 0.19 & $-5.20 * *$ & $4.66^{* *}$ & $0.41^{*}$ & $0.27^{*}$ & 0.18 & 1.46 & $0.06^{*}$ & $2.42 * *$ \\
\hline $\begin{array}{l}\text { PUNJAB UPMA X } \\
\text { FLORADADE }\end{array}$ & $4.92 * *$ & $1.97^{* *}$ & -0.50 & -1.77 & 1.07 & -0.09 & $-0.60^{* *}$ & 0.25 & 0.84 & 0.05 & 0.34 \\
\hline $\begin{array}{l}\text { PUNJAB UPMA X } \\
\text { KASHI SHARAD }\end{array}$ & $-4.75^{*}$ & -0.20 & 0.36 & 0.47 & 0.19 & 0.30 & 0.05 & $0.48^{*}$ & -0.08 & -0.02 & -0.66 \\
\hline $\begin{array}{l}\text { PUNJAB UPMA X } \\
\text { AZAD T-5 }\end{array}$ & -0.17 & $-1.77 * *$ & 0.14 & 1.30 & -1.26 & -0.21 & $0.56^{* *}$ & $-0.72 * *$ & -0.77 & -0.02 & 0.32 \\
\hline $\begin{array}{lcc}\text { PANT } & \text { T-3 } & \text { X } \\
\text { FLORADADE } & \end{array}$ & 2.63 & $7.82 * *$ & -0.19 & $-3.98^{* *}$ & $-5.94 * *$ & $0.54 * *$ & $-0.22 *$ & $0.85^{* *}$ & 0.10 & -0.04 & $-1.85^{* *}$ \\
\hline $\begin{array}{l}\text { PANT } \quad \text { T-3 } \quad \text { X } \\
\text { KASHI SHARAD }\end{array}$ & -1.27 & $-4.13 * *$ & 0.46 & $2.63^{*}$ & 0.64 & 0.14 & -0.02 & -0.12 & -0.53 & 0.01 & $1.35 * *$ \\
\hline $\begin{array}{l}\text { PANT } \quad \text { T-3 } \\
\text { AZAD T-5 }\end{array}$ & -1.35 & $-3.70^{* *}$ & -0.27 & 1.35 & $5.31 * *$ & $-0.68 * *$ & $0.24 *$ & $-0.72 * *$ & 0.43 & 0.02 & 0.50 \\
\hline $\begin{array}{ll}\text { H-86 } & \mathrm{X} \\
\text { FLORADADE } & \end{array}$ & 0.66 & 1.16 & -0.27 & 1.73 & -0.76 & -0.29 & 0.12 & -0.11 & -0.14 & 0.00 & -0.17 \\
\hline $\begin{array}{l}\text { H-86 X KASHI } \\
\text { SHARAD }\end{array}$ & $5.18^{* *}$ & -0.27 & -0.40 & -0.75 & 1.21 & 0.17 & 0.00 & -0.06 & -0.22 & -0.01 & -0.24 \\
\hline H-86X AZAD T-5 & $-5.84 * *$ & -0.88 & 0.67 & -0.97 & -0.44 & 0.12 & -0.12 & 0.17 & 0.36 & 0.01 & 0.41 \\
\hline $\begin{array}{l}\text { SELECTION-7 } \mathrm{X} \\
\text { FLORADADE }\end{array}$ & 0.73 & $1.54^{*}$ & -0.68 & 1.31 & $4.50^{*}$ & -0.32 & 0.07 & -0.06 & 0.04 & 0.03 & 0.47 \\
\hline $\begin{array}{l}\text { SELECTION-7 } \mathrm{X} \\
\text { KASHI SHARAD }\end{array}$ & 1.05 & 0.08 & -0.18 & 1.93 & -1.09 & 0.17 & 0.16 & -0.06 & 0.32 & -0.03 & $-0.78^{* *}$ \\
\hline $\begin{array}{l}\text { SELECTION- } 7 \mathrm{X} \\
\text { AZAD T-5 }\end{array}$ & -1.78 & -1.62 & 0.86 & $-3.24 *$ & -3.41 & 0.14 & $-0.23^{*}$ & 0.12 & -0.36 & -0.01 & 0.31 \\
\hline $\begin{array}{lrr}\text { NDTVR } & -60 & X \\
\text { FLORADADE } & \end{array}$ & -0.07 & $-5.49^{* *}$ & -0.96 & 2.29 & 0.69 & -0.28 & $0.23^{*}$ & -0.10 & $1.84^{*}$ & 0.03 & $1.10^{* *}$ \\
\hline $\begin{array}{l}\text { NDTVR-60 X } \\
\text { KASHI SHARAD }\end{array}$ & 2.32 & $2.00^{* *}$ & $1.62^{*}$ & -2.37 & -0.15 & 0.17 & $-0.22 *$ & -0.06 & -0.42 & -0.05 & -0.26 \\
\hline $\begin{array}{l}\text { NDTVR } \\
-60 \text { XFAZAD T-5 }\end{array}$ & -2.25 & $3.49^{* *}$ & -0.66 & 0.09 & -0.54 & 0.11 & -0.02 & 0.15 & -1.41 & 0.02 & $-0.85^{* *}$ \\
\hline $\begin{array}{l}\text { FLA } 7171 \mathrm{X} \\
\text { FLORADADE }\end{array}$ & -0.37 & $-4.49^{* *}$ & 0.27 & -0.58 & 0.56 & -0.11 & -0.03 & -0.08 & $-2.59 * *$ & -0.03 & $-1.24^{* *}$ \\
\hline $\begin{array}{l}\text { FLA7171 X } \\
\text { KASHI SHARAD }\end{array}$ & 2.90 & 0.83 & 0.56 & -1.20 & -2.56 & 0.17 & $0.20^{*}$ & -0.06 & 0.22 & 0.03 & $1.07^{* *}$ \\
\hline $\begin{array}{l}\text { FLA7171 X AZAD } \\
\text { T- } 5\end{array}$ & -2.53 & $3.66^{* *}$ & -0.83 & 1.79 & 2.00 & -0.06 & -0.17 & 0.13 & $2.37^{*}$ & 0.00 & 0.17 \\
\hline $\begin{array}{l}\text { KASHI AMRIT X } \\
\text { FLORADADE }\end{array}$ & -2.77 & 0.93 & 0.26 & -0.73 & 2.98 & -0.27 & -0.10 & -0.09 & 0.02 & $-0.05^{*}$ & 0.45 \\
\hline $\begin{array}{l}\text { KASHI AMRIT X } \\
\text { KASHI SHARAD }\end{array}$ & 0.45 & 1.23 & -0.71 & -1.91 & -0.63 & 0.17 & $0.22 *$ & -0.06 & 0.78 & $0.05^{*}$ & 0.38 \\
\hline $\begin{array}{l}\text { KASHI AMRIT X } \\
\text { AZAD T-5 }\end{array}$ & 2.32 & $-2.16^{* *}$ & 0.46 & $2.64^{*}$ & -2.35 & 0.09 & -0.11 & 0.14 & -0.79 & 0.00 & $-0.83^{* *}$ \\
\hline CD 95\% & 3.60 & 1.33 & 1.31 & 2.63 & 3.77 & 0.41 & 0.19 & 0.39 & 1.79 & 0.05 & 0.58 \\
\hline CD 99\% & 4.78 & 1.76 & 1.74 & 3.49 & 5.02 & 0.53 & 0.25 & 0.52 & 2.37 & 0.06 & 0.77 \\
\hline
\end{tabular}


Table 4. Top five specific combinations for total yield per plant on the basis of per se, gca effect of parents and showing desirable significant sca effects for other traits

\begin{tabular}{lcccc}
\hline \multicolumn{1}{c}{ Crosses } & Per se & gca effects of parents involved & sca effects & Desirable sca effect for other traits \\
\hline Punjab Upma X Azad T-5 & 4.51 & (G) $0.20^{* *} \mathrm{X}-0.06^{*}(\mathrm{P})$ & $0.56^{* *}$ & DF \\
CO-3 X Floradade & 4.56 & (G) $0.37^{* *} \mathrm{X}-0.02(\mathrm{P})$ & $0.40^{* *}$ & TSS \\
CO-3 X Azad T-5 & 3.87 & $(\mathrm{G}) 0.37^{* *} \mathrm{X}-0.06^{*}(\mathrm{P})$ & $0.27^{*}$ & PH,AFW,FC,TA,LP \\
Pant T-3 X Azad T-5 & 4.37 & (G) $0.38^{* *} \mathrm{X}-0.06^{*}(\mathrm{P})$ & $0.24^{*}$ & DF,AFW, \\
NDTVR -60 X Floradade & 3.72 & (P)-0.30**X-0.02(P) & $0.23 *$ & AFW,AC,LP \\
\hline
\end{tabular}

The crosses Punjab Upma X Azad T-5, CO-3 X Floradade, CO-3 X Azad T-5 and Pant T-3 X Azad T-5 resulted from good X poor, good X poor, good X poor, good X poor and poor X poor combining parents, also these crosses having higher per se performance. This might be due to additive $\mathrm{X}$ dominance type of interaction with epistasis gene action and non fixable genetic component for total yield per plant. NDTVR -60 X Floradade combination gives poor X poor GCA effect due to non-additive gene interaction and non-fixable genetic component for total yield per plant. This indicated possibly to obtained desirable transgressive segregants and hybrid vigour from such crosses by adopting cycle selection or biparental breeding programme.

\section{Conclusions}

The best cross combinations were Punjab Upma X Azad T-5 (for yield per plant), CO-3 X Kashi Sharad (for number of fruit per plant), Pant T-3 X Azad T-5 (for average fruit weight) and NDTVR -60 X Floradade (for earliness). Maximum desirable traits showed significantly CO-3 X Azad T-5. Also the tomato genotype Azad $\mathrm{T}-5$ proved to be the best general combiner for yield and yield components.

\section{References}

Biswas, M. K., Mondal, M. A. A., Hossain, M., \& Islam, R. (2005). Selection of suitable parents in the development of potato hybrids in Bangladesh. Chinese Potato J., 19, 193-197.

Dhaliwal, M. S., Singh, S., Cheema, D. S., \& Singh, P. (2004). Genetic analysis for important fruit characters of tomato by involving lines possessing male sterility genes. Acta Hort., 637, 123-131.

Duhan, D., Partap, P. S., Rana, M. K., \& Dahiya, M. S. (2005). Heterosis study for quality characters in a line x tester set of tomato. Haryana J. Hort. Sci., 34, 371-375.

Griffing, B. (1956). Concept of general and specific combining ability in relation to diallel system. Aust. J. Biol. Sci., 9, 463-493.

Hannan, M. M., Ahmed, M. B., Roy, U. K., Razvy, M. A., Haydar, A., Rahman, M. A., ... Islam, R. (2007). Heterosis, combining ability and genetics for brix \%, days to first fruit ripening and yield in tomato (Lycopersicon esculentum Mill.). Middle- East J. Scientific Res., 2(3-4), 128-131.

Indian Horticulture Database. (2011). National Horticulture Board, Department of Agriculture and cooperation, Government of India. Retrieved from www.nhb.gov.in

Kempthorne, O. (1957). An Introduction to Genetic Statistics. New York: John Wiley and Sons Inc.

Khan, M. A., Cheema, K. L., Masood, A., \& Sadaqat, H. A. (1991). Combining ability in cotton (Gossypium hirsutum L.). J. Agric Res., 29, 311- 318.

Mahendrakar, P. (2004). Development of F1 hybrids in tomato (Lycopersicon esculentum Mill.). Uni. Agric. Sci. Dharwad (India).

Ortiz, R., \& Golmirzaie, A. M. (2004). Combining ability analysis and correlation between breeding values in true potato seed. Plant Breeding, 123, 564-567. http://dx.doi.org/10.1111/j.1439-0523.2004.00941.x

Panse, V. G., \& Sukhatme, P. V. (1967). Statistical Methods for Agricultural Workers (II Edn.), ICAR, New Delhi.

Premalakshme, V., Thangaraj, T., Veeraranathatham, D., \& Arumagam, T. (2006). Heterosis and combining Ability analysis in tomato (Solanum lycopersicom Mill.). Wettsd. For yield and yield contributing traits. Vegetable Science, 33(1), 5-9. 
Saleem, M. Y., Asghar, M., Ahsanul, M. H., Rafique, T., Kamran, A., \& Khan, A. A. (2009). Genetic analysis to identify suitable parents for hybrid seed production in tomato (Lycopersicon esculentum Mill). Pakistan J. Bot., 41(3), 1107-1116.

Simon, J. A. (1992). Vitamin c and cardiovascular disease: A review. J. Am. Coll. Nutr., 11, 107-125.

Singh, S. P., Thakur, M. C., \& Pathania, N. K. (2010). Reciprocal cross differences and combining ability studies for some quantitative traits in tomato (Lycopersicon esculentum Mill.) under mid hill conditions of Western Himalayas. Asian Journal of Horticulture, 5(1), 172-176.

Sprague, G. F. (1966). Quantitative genetics in plant improvement. In K. J. Frey (Ed.), Plant Breeding (pp. 315-354). Ames, IA, USA: Iowa State University Press.

Yaqoob, M., Hassan, G., Mahmood, G., \& Shah, N. H. (1997). Combining ability studies for some quality traits in cotton (Gossypium hirsutum L.). J. Pure Appl Sci., 16, 47-50. 